\title{
Life Threatening Perforation of Pacemaker Atrial Lead through the Right Atrium and the Right Lung
}

\begin{abstract}
We report the case of a 65 - year old female patient who was urgently admitted to our institution with hemothorax after pacemaker implantation. The bichamber pacemaker had been implanted one week before admission and the active fixation lead caused perforation of the right atrial wall and penetration of the right lung. Transthoracic echocardiography demonstrated a minimal cardiac effusion but chest X-Ray and computed Tomography (CT) visualised the atrial screw helix outside the atrial wall penetrating through the right lung middle lobe causing hemothorax. After median sternotomy and cut-off of the atrial lead screw helix, we sew the perforated area of the right atrium and the penetrated right lung, sucking away the bloody pleural effusion before closing the thorax. Three weeks later, the cut off atrial lead was extracted through the right subclavian vein and a new one was positioned and fixed again. During the hospital admission the patient was well and free of any symptoms and troubles.
\end{abstract}

Keywords: Atrial pacing lead dislodgment; Right atrium perforation; Right lung penetration

\section{Introduction}

Cardiac pacing is a proven therapy of cardiac conduction disturbances and an effective treatment modality in many cardiac arrhythmias. Implantable cardiac defibrillators (ICDs) are particularly beneficial for selected groups of patients with a history of severe ventricular arrhythmias or at a high risk of sudden cardiac death. Despite their potential lifesaving properties, both pacemakers and ICDs are associated with a number of complications. The most frequent include pneumothorax; myocardial perforation; lead malposition, displacement or fracture due to excessive manipulation of the device by the patient (Twiddler's syndrome) [1]. Another group of complications comprises infections: from the pacemaker pocket to bacterial endocarditis with its origin on leads or the tricuspid valve, failure of leads to pace and/or sense appropriately, erosion of the pacemaker site or its leads, and thrombotic events with the most frequently observed subclavian vein thrombosis [2]. Bleeding complications with anticoagulation and antiplatelet therapy are not uncommon. Studies have reported overall lead perforation rates after pacemaker implantation to be $0.1-0.8 \%$, and after ICD placement-0.6-5.2\% [3]. The highest reported rate of perforation, based on autopsy, was $27 \%$ for patients with atrial leads [4].

There are no uniform classifications accounting for the complication. It may be acute (developing during the first $24 \mathrm{~h}$ after implantation), sub acute (up to 1 month) or chronic. Another classification system divides perforations into early (occurring during the first month after placement) and late [5]. Although the occurrence of complications of implantable pacemakers is low, there have recently been several reports of cardiac perforations from the atrial lead. We, too, describe cardiac perforation and lung penetration with this lead in one of our patients one week after pacemaker implantation.

\section{Case Presentation}

A 65-year old woman was urgently admitted in our institution with hemothorax one week after transvenous bichamber pacemaker implantation. The right- sided ventricular lead was successfully implanted but the right atrial lead was dislocated despite active fixation. The patient showed an impaired circulation with respiratory problems but no arrhythmias. The postoperative pacing control showed a dysfunction of the right atrial lead. A routine chest X-ray and computer tomography showed no signs of pneumothorax but pleural and pericardial effusions (Figure 1 \& 2).

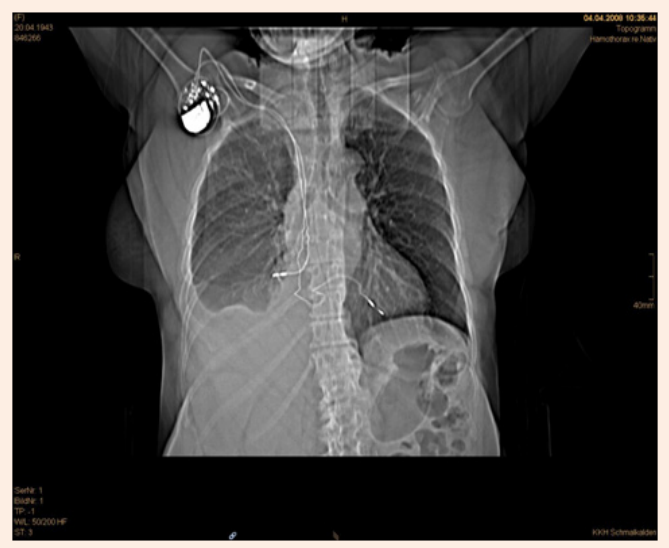

Figure 1: Thorax CT depicting the atrial lead penetrating the right lung and the combined pleural effusion.

Laboratory examination showed a low haemoglobin level while the other values were in normal rage. Four hours after admission the patient developed more chest pain, respiratory fatigue and became hypotensive. Emergency surgical exploration was decided and performed with median sternotomy. After sternotomy and heart exploration $300 \mathrm{ml}$ bloody pericardial effusion could be extracted. A perforation was found in the middle of the right atrium from the lateral side, with the dislocated lead perforating the pericardium and causing a right lung penetration (Figure 3-5). The lead was pulled out of the right atrium about 
$10 \mathrm{~cm}$ and cut off. After invagination of the cut off atrial lead in the right atrium the perforated atrium was sewn using a pursestring suture (Prolene 5x0 BB). After opening and exploration of the right pleura $300 \mathrm{ml}$ fixed haematoma and $700 \mathrm{ml}$ bloody pleural effusion were extracted. Subtle inspection of the right lung and sewing of the injury was performed. Chest closure was then performed after placement of two drainage tubes, one retro - cardiac and one right pleural (Figure 6). The patient had a normal recovery without any complications. The patient was discharged after eight days and was planned for a later atrial lead implantation in the future.

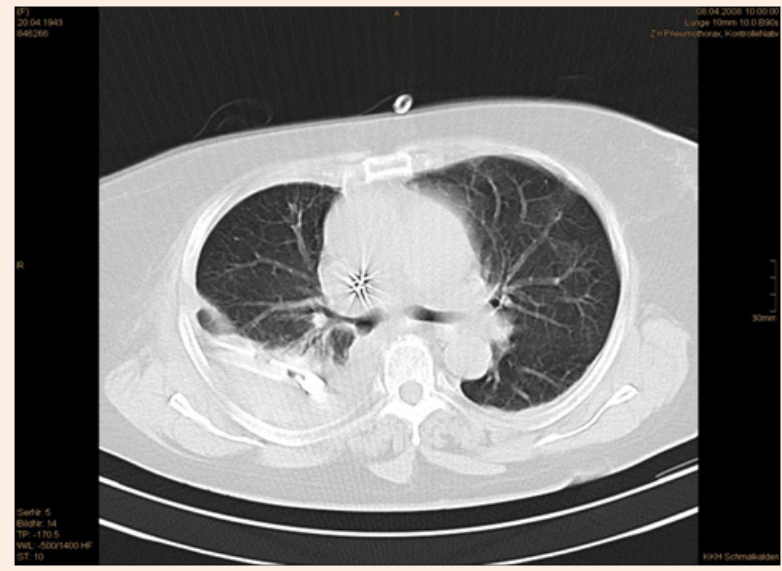

Figure 2: Thorax CT showing the atrial lead, the pleural effusion (right) and the drainage tube.

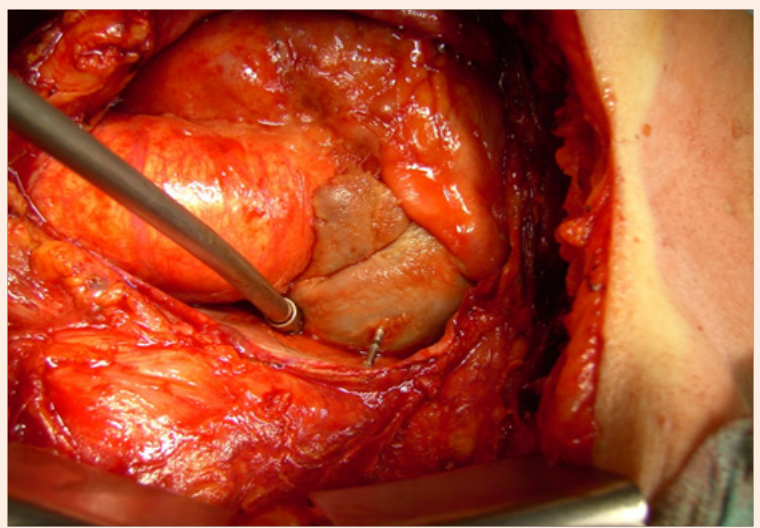

Figure 3: Perforation of the right atrium with the right atrial lead.

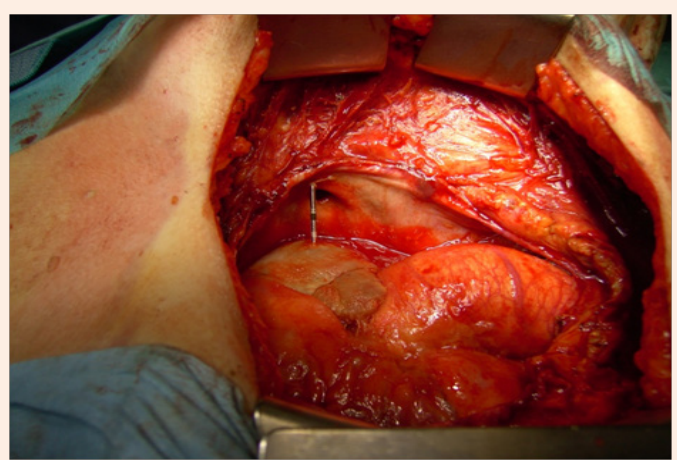

Figure 4: Perforation of the right lateral side of the pericard.

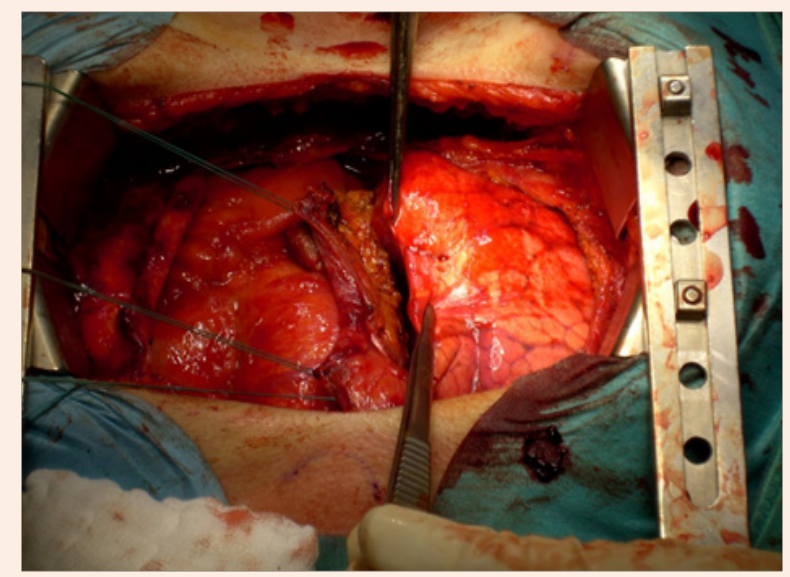

Figure 5: Penetration of the right lung before sewing.

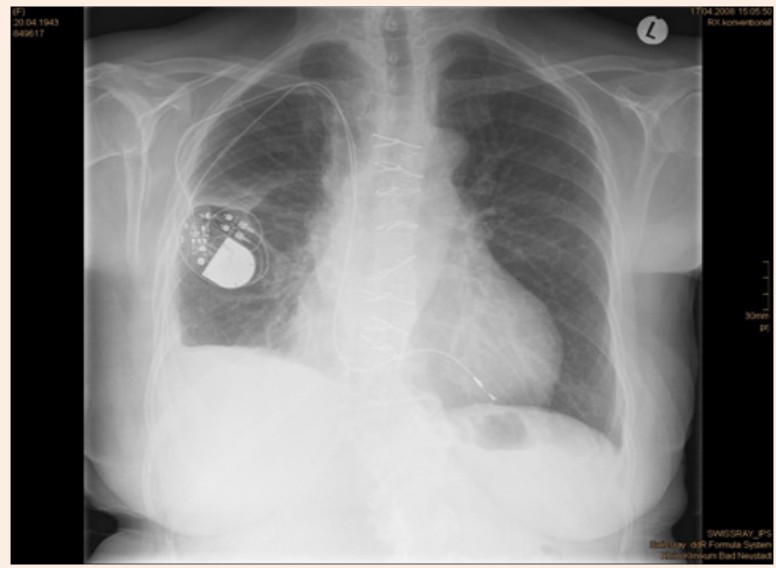

Figure 6: Plain chest X-ray after the operation.

\section{Discussion}

Iatrogenic injuries can occur in all medical sub disciplines, and interventional procedures such as pacemaker implantation are relatively often associated with such complications with various clinical images [6-8]. In this paper, we are presenting an interesting case of a 65-year old female patient, who was admitted to our department, one week after the initial procedure. What makes this case report interesting is in our opinion, the following:

a. Not only the atrial wall, but also the pericardium and the middle right lung lobe were affected,

b. The clinical image was to a great extend defined by the pleural and pericardial effusions, which finally deteriorated the clinical status of the patient and led her to the hospital.

In regard to the mechanism(s) that led to the perforation we believe that a combination of exaggerated torsion of the atrial lead helix screw during placement [9] and the existence of a thin atrial wall allowed the perforation to occur. The fact that atrial perforations were more common type than ventricular is not surprising. The right atrial wall is very thin, averaging only $2 \mathrm{~mm}$ in thickness, while the right ventricular wall is twice as thick [10]. Therefore logically one would anticipate a higher risk of atrial wall perforation. 
Symptoms, signs and changes of pacing parameters depend on the location of the lead tip. It can be located in the pericardium, mediastinum, pleural space, lung, chest muscles and even in the abdomen. Consequently, the most commonly observed symptoms include chest pain, dyspnoea, syncope, abdominal pain, muscle or diaphragm stimulation and hiccups. The haemodynamic status depends predominantly on the development of pericardial effusion. Cardiac tamponade leads to hypotension, shock or even cardiac arrest, and requires lifesaving intervention, usually openchest surgery. Perforated electrodes also frequently lead to pacing and sensing failures. In general, the capture threshold will be increased, whereas the sensing threshold will be reduced.

The most frequently reported predictors of lead perforation are temporary leads, steroid use, active fixation leads, low body mass index $\left(<20 \mathrm{~kg} / \mathrm{m}^{2}\right)$, older age, female gender and concomitant anticoagulation therapy $[11,12]$. Temporary leads are typically stiffer than permanent leads, thus elevating the risk of myocardial perforation especially in the case of recent or acute myocardial infarction. Lead type may also increase the risk of perforation: it occurs more frequently with atrial leads, active fixation systems, ICD leads, leads with a small diameter or a small tip surface - as well as excessive length of the electrode [13].

Patients with the above symptoms should be investigated to evaluate the risk of lead perforation. Pacemaker system interrogation, echocardiography, chest radiography and computed tomography (CT) scanning can be very helpful to either prove or rule out the complication. Capture and sensing threshold values should be compared against previous results. Although inappropriate pacemaker function may indicate a possibility of perforation or intracardiac leads migration, normal pacemaker function does not exclude the complication. Crucial for the diagnosis of perforation is visualization of the lead and its tip. Chest X-ray is a technique used to compare the lead tip position and lead curvature with previous results. The diagnosis of perforation is certain if the lead is outside the cardiac silhouette. It can also be helpful to evaluate extracardiac complications such as pleural or pericardial effusion and pneumothorax. Transthoracic echocardiography is a very simple, bedside tool which makes it possible to assess electrode location. It is sufficient for proving diagnosis in most cases and very helpful for monitoring pericardial effusion dynamics. Computed tomography scan proves very valuable when echocardiography and X-ray test are not diagnostic. Consequently, CT scan is currently a gold standard in the diagnosis of lead perforations [14], even though the technique also has its limitations. The star artifact is a well-known artifact related to the imaging of metal implants. Surrounding the electrode tip, it sometimes makes it difficult to precisely identify the lead tip.

Late perforations are often asymptomatic and characterized by a very low rate of cardiac tamponade or death [15]. The finding of a late perforation may represent an asymptomatic acute perforation or a true late perforation. Because the right heart is a low-pressure system, a perforation may be sealed by a combination of muscle contraction and fibrosis over the lead, resulting in no sequelae. The rate of unrecognized asymptomatic perforations is much higher than symptomatic and riches $15 \%$ [10]. Patient management strategy should depend on the symptoms, pericardial effusion and haemodynamic status. In the case of haemodynamic instability, rapid progression of pericardial effusion, surgical management (open chest surgery) seems to be the best treatment option. In other cases, in stable conditions, simple direct traction with or without percutaneous dilators can be considered, though under close echocardiographic monitoring and with a surgical backup.

During the postprocedural period, closed haemodynamic and echocardiographic monitoring is mandatory because delayed retamponade could develop (especially when the surgeon could not identify the location of cardiac muscle perforation. In cases of acute or sub acute lead perforation the implantation of a new pacemaker might be unnecessary [16]. Fortunately, the prompt and adequate surgical treatment that was applied to the patient allowed her stabilization, so that she could be discharged in a good general status 8 days after the operation. In this case the ventricular lead was protective for the patient as the pace maker system was not extracted, and we have seen it will be better to avoid the risk of trauma and infection by implantation of a new atrial lead at the same setting of this emergency operation.

\section{Conclusion}

An augmented degree of clinical suspicion should exist for all physicians dealing with similar patients, as a retarded complication in form of a pleural or pericardial effusion on the base of atrial and lung perforation may suddenly deteriorate the patient's clinical condition, even days after the original operation.

\section{References}

1. Burney K, Burchard F, Papouchado M, Wilde P (2004) Cardiac pacing systems and implantable cardiac defibrillators (ICDs): a radiological perspective of equipment, anatomy and complications. Clin Radiol 59(8): 699-708.

2. Ellenbogen KA, Wood MA, Shepard RK (2002) Delayed complications following pacemaker implantation. Pacing Clin Electrophysiol 25(8): 1155-1158.

3. Khan MN, Joseph G, Khaykin Y, Ziada KM, Wilkoff BL (2005) Delayed lead perforation: a disturbing trend. Pacing Clin Electrophysiol 28(3): 251-253.

4. Piekarz J, Lelakowski J, Rydlewska A, Majewski J (2012) Heart perforation in patients with permanent cardiac pacing -personal observations. Arch Med Sci 8(1): 70-74.

5. Ishikawa K, Cida K, Taniguchi T (1999) Myocardial perforation and/or penetration by a permanent endocardial electrode of the pacemaker in autopsy cases. J Arrhythmia 15: 29-44.

6. Sarko JA, Tiffany BR (2000) Cardiac pacemakers: evaluation and management of malfunctions. Am J Emerg Med 18(4): 435-440.

7. Binder T, Domanovits H, Berr T, Laggner A (1995) Complete generator extrusion as a cause of pacemaker dysfunction. Am J Emerg Med 13(6): 670-671.

8. Green SM (1989) Pacemaker electrode perforation of the myocardium: an unusual etiology for recurrent abdominal pain. Am J Emerg Med 7(2): 180-184.

9. Sivakumaran S, Irwin ME, Gulamhusein SS, Senaratne MP (2002) Postpacemaker implant pericarditis: incidence and outcomes with active- fixation leads. Pacing Clin Electrophysiol 25(5): 833-837.

10. Hirschl DA, Jain VR, Spindola-Franco H, Gross JN, Haramati LB (2007) Prevalence and characterization of asymptomatic pacemaker and ICD lead perforation on CT. Pacing Clin Electrophysiol 30(1): 28-32. 
11. Hermanides R, Ottervanger J, Elvan A, Ramdat Misier A (2009) Lifethreatening perforation of a defibrillation lead. Neth Heart J 17(3): 113-114.

12. Sterliński M, Przybylski A, Maciąg A, Syska P, Pytkowski M, et al. (2009) Subacute cardiac perforations associated with active fixation leads. Europace 11(2): 206-212.

13. Rydlewska A, Małecka B, Zabek A, Klimeczek P, Lelakowski J, et al. (2010) Delayed perforation of the right ventricle as a complication of permanent cardiac pacing - is following the guidellines always the right choice? Non-standard treatment - a case report and literature revive. Kardiol Pol 68(3): 357-361.
14. Henrikson CA, Leng CT, Yuh DD, Brinker JA (2006) Computed tomography to assess possible cardiac lead perforation. Pacing Clin Electrophysiol 29(5): 509-511.

15. Polin GM, Zado E, Nayak H, Cooper JM, Russo AM, et al. (2006) Proper management of pericardial tamponade as a late complication of implantable cardiac device placement. Am J Cardiol 98(2): 223-225.

16. Laborderie J, Barandon L, Ploux S, Deplagne A, Mokrani B, et al. (2008) Management of subacute and delayed right ventricular perforation with a pacing or an implantable cardioverter-defibrillator lead. Am J Cardiol 102(10): 1352-1355. 